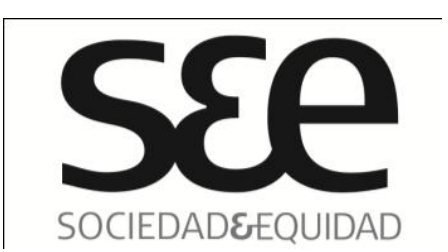

\title{
Análisis discursivo de la construcción identitaria femenina y masculina en fotonovelas de Corín Tellado
}

\author{
Nombre: $\quad$ Marcela Amaya García (*) \\ Universidad: Universidad Católica Silva Henríquez \\ Ciudad: $\quad$ Santiago \\ País: Chile \\ Correo:_mamayagarcia@gmail.com
}

Resumen

Esta investigación se centra en el análisis discursivo de la construcción de la identidad femenina y masculina en dos fotonovelas de Corín Tellado, siendo el principal objetivo identificar la producción y reproducción en el discurso de los estereotipos de la época. Siguiendo los planteamientos de van Dijk se entiende por reproducción discursiva de la dominación “(...) un acceso preferente al texto y al contexto, que se toman como base o recurso de poder, comparable a recursos sociales (...)" (van Dijk, 1997b: 19). Los resultados muestran cómo se expresan, representan y se legitiman en el discurso formas de desigualdad entre géneros propias del sistema patriarcal del período.

\section{Palabras claves}

Identidad, estereotipo, discurso, fotonovelas, análisis crítico del discurso.

\section{Discourse analysis of male and female identity construction in fotonovelas of Corin Tellado}

This research in centered on the analysis discursive of the construction of the femenine and masculine identity on two Corin Tellado's photonovels. Being the principal objetive to identify the production and reproduction on the speech of the stereotypes of the period. Following the expositions of Van Dijk, it's understood by discursive reproduction of the denomination "(...) a preferential access to the text and the context, they take as base or resourse of power, comparable to social resourse (...)" (van Dijk 1997b:19). The results show how they express, represent and the forms on inequality are legetimized in the speech between generes proper of the patriarcal commanding system in the above mentiones period.

\section{Keywords}

Identity, stereotype, speech, photonovels, critical discourse analysis.

(`)La autora es Dra. (c) Ciencias de la Educación, Magíster en Linguística y Periodista. Sus líneas de investigación son: Análisis Crítico del Discurso, Lingüística Aplicada, Didáctica de la Lengua. 


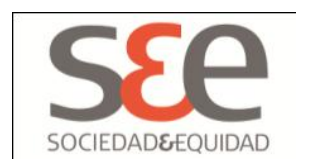

\section{Introducción}

Mientras autores como Álvarez (2001), Charadeau y Maingenau (2005), Gee (1999), Jaworski y Coupland (1999) y, Pomerantz y Fehr (2001) han proporcionado importantes aportes teóricos relativos al discurso, sus tipologías y técnica de abordaje conocida como análisis del discurso, otros han puesto sus esfuerzos en un acercamiento al texto desde una perspectiva centrada en el análisis crítico del discurso. Uno de ellos es van Dijk (1997a, 1997b, 2003), quien ve en este un método a través del cual es posible develar los abusos de poder y las eventuales diferencias en el acceso a la producción de discursos en tanto reproductor de estrategias de dominación.

Aún cuando una multiplicidad de estudios críticos han analizado el papel de los medios de comunicación de masas en Chile en diferentes contextos, por ejemplo, Vera, Aravena y otros (2004) identificaron la "Calidad de la Información Periodística. Investigación sobre Diarios Chilenos: aspectos Teóricos y Metodológicos" y, Blanco y Silva (1998) buscaron "La Legitimación del Discurso del Placer de la Sexualidad Femenina en dos Suplementos y una Revista, desde la Perspectiva del Análisis Crítico del Discurso"; lo cierto es que aún existe una brecha en el conocimiento respecto a la forma de construcción discursiva de la identidad femenina y masculina en medios de comunicación masivos que han sido considerados fenómenos socioculturales, tal es el caso de las fotonovelas de Corín Tellado.

El presente artículo surge como una primera aproximación para llenar ese vacío, que presenta en su parte inicial el contexto socio-cultural en que tiene lugar el fenómeno en estudio. Posteriormente, se detallan algunos aspectos teóricos en torno al concepto de discurso, análisis crítico del discurso y novela rosa. Más adelante, se da cuenta de los procedimientos metodológicos empleados en la investigación. Le sigue una descripción de los resultados que muestran cómo el discurso en las fotonovelas de Corín Tellado legitima y reproduce las desigualdades identitarias de género imperantes en aquellos años $y$, finalmente, en las conclusiones se hace una síntesis de los principales hallazgos y se pone en perspectiva la realización de futuros trabajos.

\section{Contexto socio-cultural}

Esta investigación es menester enmarcarla tanto en un contexto internacional como local, puesto que se trató de una fotonovela de tiraje mundial, cuyo formato fue incorporado al mercado interno, llegando a ser un referente en entre las mujeres de la época.

Contexto Internacional: “Corín Tellado” como mass media

La zaga de novelas Corín Tellado nace en España, en 1946, cuando su autora Socorro Tellado López se incorpora al staff de escritores de Editorial Bruguera, siendo el primer título publicado "Atrevida". Sus novelas se caracterizaron por ser cortas, de no más de cien páginas, de escenarios románticos donde tenían lugar historias de amor y desamor entre los protagonistas, los que por norma terminaban convertidos en un feliz matrimonio. 


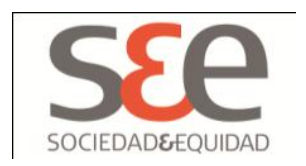

En 1951, debido al éxito alcanzado en el Viejo Continente, la "Revista Vanidades" decide expandir Corín Tellado por Hispanoamérica. De esta manera, el tiraje de la revista crece de 16 mil a 68 mil ejemplares cada quincena.

Posteriormente, a fines de 1966 la industria se expande con la salida al mercado de la colección de libros de Corín Tellado (fotonovelas de circulación quincenal). Pero es en 1970 cuando "Socorrín" -como fue conocida la autora- instala en la pantalla gigante sus dramas de amor por medio de la película "Tengo que abandonarte".

En 1977 Corín Tellado llega a la radio con la serie radiofónica "Lorena" y, en 1979 se pone a la venta una colección de novelas eróticas que firma bajo el seudónimo de "Ana Miller".

Ya entrada la década de los '80, el público objetivo del formato se diversificó hacia grupos etarios de menor edad, de esta manera con el respaldo de la Editorial Cantábrico se publican doce novelas juveniles. La televisión española (canal Tele 5), en tanto, pone en 1990 en el aire una serie de veintiséis capítulos titulada "Las grandes novelas de Corín Tellado".

Según datos publicados en el sitio en Internet de la autora, se estima que desde 1946 a la fecha se "[han] publicado unos 4.000 títulos y vendido más de 40 millones de ejemplares de sus novelas", teniendo “(...) éxito en especial en Latinoamérica donde impulsaron la creación del 'culebrón' o serial televisivo" (www.corintellado.com). Al respecto, un dato no menor es la incorporación de Corín Tellado, en 1994, al record Guiness como la escritora más leída en lengua hispana.

Lo anterior cobra relevancia en función de lo que Adorno (1967) define como industria cultural, es decir, “(...) la estandarización de la cosa misma -por ejemplo la estandarización del western, familiar a cada espectador de cine-, y a la racionalización de las técnicas de distribución, y no estrictamente al proceso de producción" (Adorno, 1967: 12). "En efecto, la industria cultural es importante, como factor dominante del espíritu, hoy. Querer subestimar su influencia por escepticismo en atención a lo que transmite a los hombres, sería una ingenuidad" (Adorno, 1967: 14). En ese sentido, la propia Corín Tellado -en su web en Internet- reconoce que su estilo es producto de la censura franquista, que sólo permitió la publicación de historias de amor con bodas como final.

El punto central respecto a la industria cultural y que dice relación con las fotonovelas de Corín Tellado -como dice Adorno (1967)- es que “(...) la dominación técnica progresiva se transforma en un engaño de masas, es decir, en un medio de oprimir la conciencia. Impide la formación de individuos autónomos, independientes, capaces de juzgar y decidir conscientemente" (Adorno, 1967: 20). 


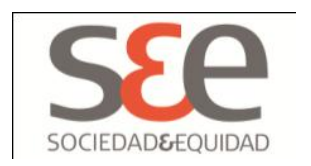

Contexto local: La realidad nacional

Blanco y Silva (1998), citando a Santa Cruz señalan que "los contenidos de las publicaciones dedicadas a las mujeres por reproducir modelos de vida distan mucho de la realidad criolla, promoviendo ideales de moda y belleza que quizá no combinen con la chilena tradicional" (Blanco y Silva, 1998: 90). Las autoras, complementando lo antes expuesto y siguiendo a Munizaga, comentan que las mujeres chilenas tienden a utilizar sus ratos de esparcimiento para aprender por medio de la lectura, por lo tanto, los medios de comunicación destinados a ellas son el mecanismo para saciar sus ganas de adquirir nuevos conocimientos.

En consecuencia, para Blanco y Silva (1998) es primordial observar la relación medios-mujer, ya que consideran que estos son bastiones en los que “(...) el predomino de la clase dominante intenta asegurar su poder" (Blanco y Silva, 1998: 90). Específicamente, en relación al período de circulación de las fotonovelas de Corín Tellado que forman parte de la muestra, las autoras plantean lo siguiente:

Durante los años del gobierno militar, se exageraron las diferencias entre hombres y mujeres, y a ellas se les asignó el rol de 'necesidad de protección', por su debilidad física e indefensión. Se asocia la feminidad a la imagen tradicional de la esposa-dueña de casa, reforzando indirectamente la principal base de sustentación de la familia patriarcal. (...) El discurso del régimen militar hacia la mujer apuntó hacia los elementos más conservadores de la ideología patriarcal, asignándole el rol prioritario de la maternidad y fomentando en ellas conductas abnegadas y obedientes, en un proceso de socialización que favoreció su adaptación a la dominación. Era un discurso eminentemente conservador y perpetuador de lo tradicional, que tendió a la mantención de la desigualdad al promover la adaptación de las realidades y relaciones autoritarias y jerárquicas que enfrentan las mujeres en sus relaciones de pareja, en sus hogares, y que ven repetidas en las historias de otras mujeres, tanto de aquellas que conocen, como las que se muestran en los medios de comunicación, [por ejemplo, Corín Tellado]

(Blanco y Silva, 1998: 68)

\section{Hipótesis}

Las fotonovelas de Corín Tellado perpetúan a través del discurso la identidad femenina y masculina de los estereotipos vigentes en esa época. Estas consideran el ideario de una sociedad patriarcal, donde la mujer cumple el rol de esposa-abnegada y el varón el de marido-protector, entre otros. 


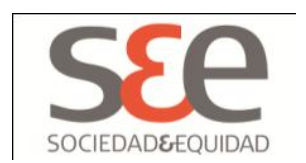

\section{Marco teórico}

Concepto de discurso y análisis crítico del discurso

En primer lugar, según Charadeau y Maingenau (2005: 179) "el discurso es una noción que se encontraba ya en la filosofía clásica, donde al conocimiento discursivo por concatenación de razones se le oponía el conocimiento intuitivo". En tanto, para Álvarez existe una polisemia entre los términos texto y discurso, lo que implica un uso indistinto de ambos. Sin embargo, precisa que mientras "el texto es una configuración lingüística. Es un conjunto de elementos lingüísticos (palabras, oraciones...) organizados según reglas estrictas; el discurso es la emisión concreta de un texto, por un enunciado determinado, en una situación de comunicación determinada" (Álvarez, 2001: 11-12).

Por su parte, van Dijk (2001) desecha la distinción entre el discurso oral y el escrito, puesto que la conversación y el texto son dos grandes manifestaciones de la lengua. Una postura un tanto similar es la que presentan Jaworski y Coupland (1999), quienes señalan que el discurso en su nivel más básico puede ser entendido como el estudio o análisis del lenguaje en uso y que involucra aspectos sociales relacionados con formaciones sociales, políticas y culturales.

Dicha concepción surgida más bien desde la pragmática, la adopta también Gee (1999) cuando se refiere al discurso. En efecto, lo relaciona directamente con el lenguaje en uso que se emplea en la construcción in situ de actividades e identidades. No obstante, determina una clara diferencia con el Discurso que entiende como la unión entre la parole y todo lo que le rodea, vale decir, la vestimenta, los gestos, los símbolos, entre otros. Sin embargo, van Dijk (1982) incorpora la noción de episodio al discurso, donde el episodio es una unidad semántica o una secuencia específica de proposiciones que deben tener coherencia local y global, mientras que un párrafo es la manifestación superficial de éste. A su vez, estas secuencias de proposiciones deben estar incluidas en las llamadas macroproposiciones en función de lo cual es posible entender la unidad total del discurso y determinar elementos claves para la comprensión de éste, tales como el tema y los tópicos. Precisamente estos, además de la cohesión y la coherencia textual, forman parte de las unidades analíticas del discurso en distintos niveles.

Respecto del análisis del discurso, Gee (1999) señala que el objetivo fundamental de quien efectúa esta clase de investigaciones es establecer relaciones entre los enunciados a fin de problematizar sobre la manera en que los seres humanos construyen los significados. Para efectuar la anterior tarea proponen una guía metodológica que incluye, a lo menos, dieciocho preguntas posibles de formular al texto. A su vez, Jaworski y Coupland (1999) sostienen que la base del análisis del discurso es el análisis funcional del lenguaje y de otros sistemas semióticos, cuya información textual es recopilada, por ejemplo, por medio de grabaciones de audio y video. 


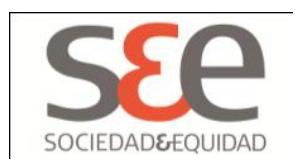

Por otro lado, van Dijk (1997a: 13-14) habla de la "ciencia del texto como nueva ciencia interdisciplinaria". En ese sentido, recalca que la ciencia del texto aspira a algo más general y abarcador: por un lado se refiere a todo tipo de textos y a los diversos contextos que le corresponden $y$, por otro, se preocupa de los procederes más bien teóricos, descriptivos y aplicados. De ahí, se deduce que el origen de una nueva ciencia dedicada al análisis más general de textos se parangona con evoluciones de otras disciplinas y que, con ello, representa la prosecución consecuente de una tendencia a estudiar el uso de la lengua y la comunicación de manera interdisciplinaria. También Pomerantz y Fehr (2001), citando a Coulthard y Montgomery, apuntan que el análisis del discurso nace con el objetivo de dar respuestas sobre elementos discursivos que las oraciones.

Por otra parte, una aproximación al discurso desde una perspectiva multidisciplinaria es la que ofrece el análisis crítico del discurso (ACD), definido por (Fairclough, 2001: 387) como “(...) el análisis de los aspectos lingüísticos y semióticos de los procesos y de los problemas sociales", siendo -a juicio de este autor- su principal característica "[tomar] partido a favor de los grupos oprimidos en contra de los grupos dominantes y (manifestar) abiertamente la vocación emancipadora que la motiva" (Fairclough, 2001: 368). En ese sentido, Farías (2006) señala que el ACD es una vertiente del análisis del discurso que permite, entre otros, develar ideologías, poner en el tapete temas como el racismo, la discriminación de la mujer y establecer relaciones de poder.

Siguiendo esta línea de argumentación, Burgueño (2006) recalca que través de esta perspectiva teorética es posible evidenciar los diferentes mecanismos de producción de significado en un orden establecido. De modo, que es un método que facilita la localización de los "procesos de exclusión" al interior de la sociedad, tales como "el tabú del objeto, ritual de la circunstancia y derecho exclusivo o privilegio del sujeto que habla". "En toda sociedad la producción del discurso está a la vez controlada y seleccionada y redistribuida por cierto número de procedimientos que tienen por función conjurar sus poderes y peligros, dominar el acontecimiento aleatorio y esquivar su pesada y temible materialidad" (Foucault,1999: 14).

Por consiguiente, el ACD, señala Fairclough (1998), ofrece no sólo la oportunidad de efectuar una crítica al sistema ideológico como ha sido hasta hoy, sino que también dar mayor atención a temas como las relaciones sociales, la globalización del discurso, etc. Como señala van Dijk (2003: 171) "el ACD puede estar interesado en macronociones como las del poder y la dominación, pero su estudio efectivo tiene lugar en el microplano del discurso y de las prácticas sociales."

Es decir, el ACD debe hacer suyos los frecuentes cambios en la vida y en la sociedad. De manera, se plantea como el método adecuado y pertinente de aplicar en esta investigación, cuyo eje es el análisis discursivo de la construcción de la identidad femenina y masculina en dos fotonovelas de Corín Tellado en función de la producción y reproducción en el discurso de los estereotipos de la época. 


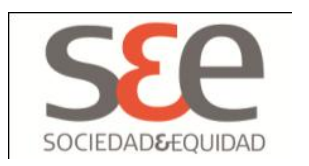

Novela Rosa, amor romántico y relaciones de género

De acuerdo a Martínez (2000: 529) la novela rosa o romántica forma parte de un subgénero literario, cuyo objetivo es “(...) la conservación tópica y arquetípica de la 'feminidad', vista y entendida desde una óptica exclusivamente patriarcal". Asimismo, la autora antes citada sintetiza como sigue las principales características del llamado "rosa".

Todo el relato [es] visto como un sistema cerrado de retroalimentación, se mueve obsesivamente alrededor de la consecución del mismo motivo narrativo: el sentimiento, el reconocimiento, la declaración del amor, la separación y la reconciliación de los enamorados. El famosísimo lzappy end puede dilatarse hasta la exasperación y el enfrentamiento entre los enamorados debe ser entendido solamente como un truco retórico para fomentar la pasión, aumentar las expectativas en el logro receptivo y en el placer del tan sabido y al mismo tiempo deseado final feliz.

(Martínez, 2000: 532)

A su vez, Martínez (2000) explica que la novela rosa se aleja de cualquier conflicto amoroso, pues busca instruir a las lectoras en la felicidad, ya que se trata de un amor fantástico y, siguiendo Beauvoir (1981) y Martínez Garrido (1995), reconoce que perpetua las diferencias de género e incentiva el consumo.

Ante el incentivo fantástico y amoroso del género rosa, no sólo se afianzan los ancestrales papeles y funciones atribuidos a la diferencia sexual y a cualquier otro tipo de diferencia, sino que además se favorece el consumo; la ropa, el calzado, el perfume, los atuendos de la seducción, entendidos como 'armas de mujer' son ayudantes imprescindibles de las protagonistas textuales y extratextuales para lograr realizar su 'sueño' y su 'locura de amor'.

(Martínez, 2000: 533)

Desde luego, una opinión similar presenta Alonso (2011: 36), quien señala que un buen ejemplo de ello eran las novelas de Corín Tellado, donde “(...) se utilizan descripciones literarias del amor completamente idealizadas y exaltadas (...)". En esta misma línea argumentativa, la autora antes referenciada agrega que el idealismo que se amalgama en este tipo de textos “(...) la imagen de las mujeres se resuelve en una mística de la feminidad entendida como debilidad y sumisión, dentro de la imagen y el esquema más tradicional. Eso significa, en primer lugar, rechazo de la soltería" (Alonso, 2011: 38). Así, la tarea principal de estas novelas es mostrar "(...) lo que tenía que ser y hacer una mujer, nombraban el orden y llamaban al orden" (Alonso, 2011, 39). Por otro 


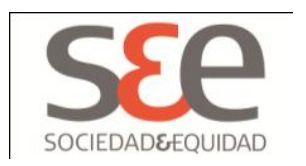

lado, explica que las novelan rosas tendían a emplear un "vocabulario lleno de metáforas guerreras y de conquista bélica y la utilización del lenguaje estaba marcado por completo por la censura, que obligaba a un amplio uso del eufemismo, fundamentalmente en lo relativo a cuestiones sexuales" (Alonso, 2011: 42).

Guerra (1989: 685) va un poco más allá, planteando que en la literatura, especialmente la rosa, se ha plasmado la imagen de una mujer ligada a la pureza como parte de un modelo patriarcal, donde ésta es mirada bajo la lupa del peligro.

La representación literaria de la sexualidad femenina, desde una perspectiva patriarcal, reproduce imaginariamente dos figuras básicas en la cultura de Occidente: la virgo intacta, signo, por excelencia, de la inocencia sexual que sacraliza la maternidad en la imagen de la Virgen María y la vagina dentata que postula la apertura a la sexualidad como un peligro de devoración. Oposición semántica que fija a la mujer en la alteridad y que simultáneamente utiliza el sujeto masculino para afianzar su propia identidad.

Al mismo tiempo, un aporte interesante sobre el amor romántico lo realiza Martínez-Plana (2004: 23), quien sostiene que este “(...) es un buen ejemplo de la cosificación de la cultura capitalista en entendidos y significados de acción. Une y condensa las siguientes contradicciones: la esfera del consumismo y de la producción, el (des) orden actual y la siempre poderosa disciplina ética religiosa, la utopía de la no existencia de clases y la dinámica de la distinción social". En este contexto, entonces, el género -dice Martínez (2007: 88)- como tal "es concebido aquí como una construcción histórico-cultural que prescribe determinadas formas diferenciales de pensar, sentir y ser para hombres y mujeres". De este modo, "lo masculino y lo femenino no sólo son construcciones sociales, sino también filtro cultural, constitución subjetiva e interpretación genérica del mundo".

Por último, cobra relevancia en relación a la novela rosa lo establecido por Blásquez (2008: 2) en cuanto a que "el amor en pareja y el amor sexual, es una de las principales formas de control social de las mujeres, pues las educa para que reproduzcan una sistema de género desigualitario basado en la entrega como necesidad amorosa".

\section{Metodología}

Los procedimientos metodológicos de esta investigación se inscriben en los planteamientos de van Dijk (1997b, 2003) en tanto análisis crítico del discurso (ACD) entendido como “(...) un enfoque especial en el análisis de discurso que se fija en las condiciones discursivas, en los componentes y en las consecuencias del abuso de poder ejercido por grupos [élite] dominantes e instituciones" (van Dijk, 1997b: 24).

Se abordó el ACD para evaluar cualitativamente sólo los aspectos textuales en las dos fotonovelas de Corín Tellado, dejando al margen de este estudio un enfoque semiótico e iconográfico de ellas. 


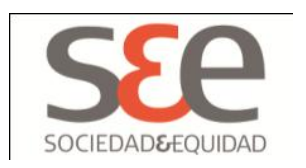

Este estudio se realizó sobre una muestra de 86 enunciados extraídos desde 512 fotogramas que forman parte de dos fotonovelas de Corín Tellado de distribución semanal tituladas "Te atreviste por Mí" y "Te vi Pasar" publicadas por la Editorial América S. A. en 1971. La selección de la muestra de revistas se efectúo en forma aleatoria, considerando que las fotonovelas en cuestión respondieran con las características propias de la tipología textual descrita en el marco teórico, vale decir, contener un relato normalmente de carácter amoroso formado por una sucesión de fotogramas u fotografías acompañadas de diálogos a través de los cuales era posible seguir el desarrollo del argumento.

Delimitada la muestra, se procedió a establecer las siguientes unidades de análisis:

1.- Construcción de la identidad femenina

2.- Construcción de la identidad masculina

3.- Construcción de la identidad femenina y masculina desde el narrador

TABLA 1. CUADRO RESUMEN CORPUS

\begin{tabular}{|c|c|c|c|c|c|}
\hline \multirow{3}{*}{$\begin{array}{l}\text { CORPUS } \\
\text { FOTONOVELA }\end{array}$} & \multicolumn{4}{|c|}{ CONS TRUCCIONDELA IDENIDAD } & \multirow{3}{*}{$\begin{array}{c}\text { TOTAL } \\
\text { ENMCAADOS } \\
\end{array}$} \\
\hline & \multicolumn{4}{|c|}{ NAFFADCR } & \\
\hline & MUER & HOMERE & MWER & HOMEFE & \\
\hline FOTONOVEA 1 & 22 & 16 & 7 & 2 & 47 \\
\hline FOTONOVEA 2 & 20 & 17 & 1 & 1 & 39 \\
\hline TOIMUES & 42 & 33 & 8 & 3 & {$[3$} \\
\hline
\end{tabular}

Creado por la autora

El registro de los textos relativos a las unidades de análisis anteriormente enumerados se efectuó en función de su representación discursiva en cada una de las fotonovelas parte de la muestra. Para facilitar el trabajo se utilizó la nomenclatura Fotonovela 1 y Fotonovela 2 en alusión a las fotonovelas "Te Atreviste por Mí" y "Te vi Pasar", respectivamente.

\section{Análisis}

Construcción de la identidad femenina

Del total de 86 enunciados analizados, 42 de ellos correspondieron a enunciados referidos al ideario identitario de la mujer. Vale decir, el 48,8 por ciento de los enunciados extraídos de 


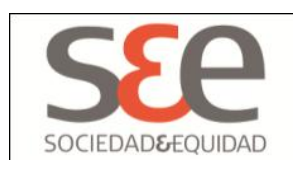

ambas revistas de Corin Tellado constituyeron alusiones al rol de la mujer y su modo de entender y enfrentar el mundo en el período en estudio (1971).

TABLA 2. CUADRO CONSTRUCCIÓN DE LA IDENTIDAD FEMENINA

\begin{tabular}{|c|c|c|}
\hline \multirow[b]{2}{*}{$\begin{array}{c}\text { CORPUS } \\
\text { FOTONOVELA }\end{array}$} & CONSTRUCCIONDE LA TDENTIDAD & \\
\hline & 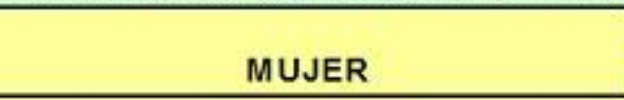 & $\begin{array}{l}\text { PORCENTAJE } \\
\text { ENUNCIADOS }\end{array}$ \\
\hline FOTONOVELA 1 & 22 & $25,50 \%$ \\
\hline FOTONOVELA 2 & 20 & $23,25 \%$ \\
\hline TOTRLES & 42 & 49.800 \\
\hline
\end{tabular}

Creado por la autora.

En la tabla 2, se aprecia que el número de enunciados relativos a la identidad femenina registrados en las dos fotonovelas son prácticamente equiparables. Mientras en la fotonovela 1 la cantidad alcanzó a 22; en la fotonovela llegó a 20.

La fotonovela 1 presenta una mujer romántica que cree en el amor, en el matrimonio, en la entrega desinteresada y profunda basada en sus sentimientos.

Ejemplo 1: "Nunca me casaría por un interés material. Tiene que ser horrible vivir junto a un hombre que no se ama".

Ejemplo 2: "Sólo nos diferenciamos en una cosa. Yo soy una sentimental y tú (...)".

Ejemplo 3: "No pienso quedarme soltera, pero deseo casarme muy enamorada".

Lo antes expuesto, también, se da en la fotonovela 2 aún cuando aquí el argumento pretende dar cuenta de una mujer más independiente, que se incorpora al mundo del trabajo. En el fondo, la protagonista de esta historia sigue buscando el amor, y por qué no el matrimonio, que finalmente encuentra.

Ejemplo 4: "No quería una secretaria, quería una amante. Y yo no sirvo para eso".

Ejemplo 5: "Te quiero, Elena... Me he enamorado de ti... quiero que seas mi esposa". 


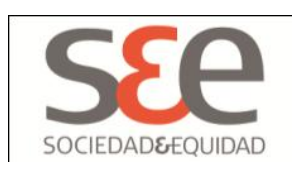

Asimismo, en las dos fotonovelas se resaltan virtudes en relación a la figura femenina, tales como la honestidad, la belleza, la humildad, la sinceridad, entre otras.

Ejemplo 6: "(...) Tiene demasiada clase".

Ejemplo 7: "No soy ambiciosa, Fernando".

Ejemplo 8: "Estás más bella que nunca y te siento tan dentro de mí, como si fueras una cosa propia".

Ejemplo 9: "He robado el dinero de tu hijo. No tengo el menor derecho a ese capital".

Ejemplo 10: "Eres demasiado ingenua, Elena. Ingenua y buena y eso te acarreará muchos disgustos en la vida".

Ejemplo 11: "Es una muchacha muy dúctil. Aprenderá pronto (...)".

Ejemplo 12: "Soy muy sincera en todo, Señor Acosta (...)".

Por otra parte, mientras que en la fotonovela 1 el discurso refleja a una mujer que se autocastiga y flagela por realizar acciones que estarían fuera de los cánones sociales de la época, entre ellos, sentirse atraída sexualmente por un hombre; en la fotonovela 2 se muestra la representación de una mujer ideal: cariñosa, hogareña, ordenada, es decir, una ama de casa.

Ejemplo 13: "Y me siento avergonzada, inmoral".

Ejemplo 14: "Quizás comience a creer en el que encuentre la mujer perfecta, o dicho de otro modo, la mujer que me satisfaga plenamente". "Pero usted se aproxima bastante al ideal".

Ejemplo 15: "(...) Me estaba haciendo falta una persona como usted. Habrá visto que mi vida es muy desordenada".

Finalmente, las mujeres de ambas historias, en especial la de la fotonovela 2, vive experiencias vicarias, aspirando a ser como otras que tienen según sus patrones más mundo, más experiencia. Sin embargo, en el discurso se terminará por cuestionar a aquellas que tienen una opción distinta al matrimonio y a los convencionalismos.

Ejemplo 16: "Me hubiera gustado ser actriz como tú".

Ejemplo 17: "Me gustaría conocerla, debe ser una mujer interesante y con experiencia para tener atrapado a un hombre como él".

Ejemplo 18: "Vivo con una amiga actriz y también lleva una vida muy desordenada". 


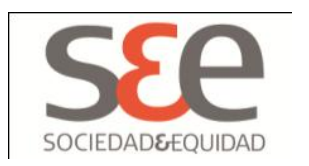

Construcción de la identidad masculina

Del total de 86 enunciados analizados, 33 de ellos correspondieron a alusiones a la identidad masculina en el discurso de las fotonovelas de Corín Tellado. Por lo tanto, el 38,36 por ciento de los enunciados estuvieron enfocados a presentar un ideario de hombre en concordancia con el contexto sociocultural y los tiempos en cuestión.

TABLA 3. CUADRO CONSTRUCCIÓN DE LA IDENTIDAD MASCULINA

\begin{tabular}{|c|c|c|}
\hline \multirow{2}{*}{$\begin{array}{c}\text { CORPUS } \\
\text { FOTONOVELA }\end{array}$} & CONS TRUCCION DE LA IDENTIDAD & \\
\hline & & PORCENTAJE \\
\hline FOTONOVELA 1 & 16 & $18,60 \%$ \\
\hline FOTONOVELA 2 & 17 & $19,76 \%$ \\
\hline TOETRES & 33 & 88,36 \\
\hline
\end{tabular}

\section{Creado por la autora.}

En la tabla 3, se muestra que de los 33 enunciados analizados existe un número casi equivalente entre ambas novelas: 16, la fotonovela 1 y 17, la fotonovela 2. Cabe recordar que una situación similar se dio con los enunciados referidos a la identidad femenina. No obstante, comparado con el número de enunciados relativos al rol de la mujer, se constata que existe una diferencia de 10 puntos porcentuales.

En las fotonovelas, por lo general, se representa a los varones viviendo en constante dicotomía, es decir, entre el amor romántico y el impulso frenado o del deseo por lo cual son condenados en el discurso. Al tiempo, que ellos se arrepienten y se culpan por su irreverente descontrol.

Ejemplo 18: "En toda mi vida no conocí un quijote como tú".

Ejemplo 19: "Todavía no sé si Javier es un sádico o un hombre terriblemente apasionado".

Ejemplo 20: "(...) Esto no te da derecho a comportarse como un salvaje".

Ejemplo 21: "(...) El tipo se ha portado como un puerco".

Ejemplo 22: "Nadie es perfecto y en algunos momentos de la vida no hay quien pueda verse libre de un ataque de locura". 


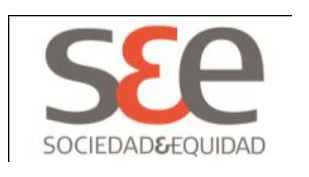

A su vez, a diferencia de lo que sucede con la identidad femenina, donde Corín Tellado destaca las virtudes de la mujer; en la construcción de la identidad masculina la autora presenta hombres bohemios, mujeriegos y desordenados, que encuentran la felicidad y el camino al lado de una esposa.

Ejemplo 23: "Nunca creí que fuese usted tan ruin, Señor Acosta, me ha decepcionado profundamente".

Ejemplo 24: "Tú... sabes que no soy... ningún canalla (...)".

Ejemplo 25: "El famoso director de televisión y cine, Sergio Acosta, se aprovecha de su posición para conquistar a las jóvenes que sueñan con un rutilante porvenir como actrices...".

Ejemplo 26: "Los hombres, cuando llegáis a cierta edad, no valéis nada si no tenéis una mujer a vuestro lado".

Por último, se ven reflejados en el discurso de las fotonovelas de Corín Tellado algunos estereotipos sobre los hombres inmersos en el imaginario colectivo.

Ejemplo 27: "(...) Todos los jefes se portan de igual modo con sus nuevas secretarias (...)".

Ejemplo 28: "En el mundo del teatro, del cine y de la televisión, se conocen tipos de todas las clases, la mayoría de los cuales sólo van con intención de llevarte a su apartamento".

Construcción de la identidad femenina y masculina desde el narrador

Del total de 86 enunciados analizados, 12 de ellos correspondieron a alusiones a la identidad femenina y masculina desde el narrador del discurso, distinguiéndose 8 referidos a la identidad de la mujer y 3 a la identidad del hombre. En porcentajes, esto equivale al 9,30 y al 3,49 por ciento del total de enunciados estudiados. 


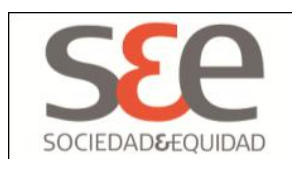

TABLA 4. CUADRO CONSTRUCCIÓN DE LA IDENTIDAD FEMENINA Y MASCULINA DESDE EL NARRADOR

\begin{tabular}{|c|c|c|c|}
\hline \multirow{3}{*}{$\begin{array}{l}\text { CORPUS } \\
\text { FOTONOVELA }\end{array}$} & \multirow{2}{*}{\multicolumn{2}{|c|}{$\begin{array}{c}\text { CONS TRUCCION DE LA IDENTIDAD } \\
\text { NARRADOR }\end{array}$}} & \multirow{3}{*}{$\begin{array}{l}\text { PORCENTAJE } \\
\text { ENUNCIADOS }\end{array}$} \\
\hline & & & \\
\hline & MUJER & HOMBRE & \\
\hline FOTONOVELA 1 & 7 & 2 & $10,46 \%$ \\
\hline FOTONOVELA 2 & 1 & 1 & $2,31 \%$ \\
\hline TOTARLES & 8 & 3 & 12.790 \\
\hline
\end{tabular}

Creado por la autora.

En la tabla 4, se aprecia que el narrador omnipresente crea y representa en el discurso con mayor continuidad las sensaciones de la mujer, puesto que se instala en ambas fotonovelas como un tercero que observa los acontecimientos y hace patente el mundo interno femenino, donde destacan los sentimientos de angustia, ansiedad, temor, miedo, tristeza, etc.

Ejemplo 29: "Recordó la humillación sufrida, su impotencia, pero no podía olvidar que cada una de las fibras de su ser vibraron en aquel momento de su vida".

Ejemplo 30: "Victoria estaba hecha un mar de dudas, sobre los verdaderos sentimientos de Javier".

Ejemplo 31: "Amaba a Javier y le dolía haber sido humillada".

Ejemplo 32: "Se sentía aturdida, desconcertada... y sin darse cuenta, se encontró en los brazos de Sergio...".

En relación a la identidad masculina, el narrador ratifica los rasgos negativos de los hombres al igual que el texto mismo. En definitiva, el narrador construye un modelo varonil reconocible en la época.

Ejemplo 33: "La vida y las costumbres de Sergio Acosta, no tenían ninguna clase de orden. Era frecuente que a las dos de la tarde, aún estuviera durmiendo".

Ejemplo 34: "Fue un beso doloroso, casi inhumano, (...) y envilecía a Javier".

Ejemplo 35: "(...) Preso de una excitación impropia en él". 


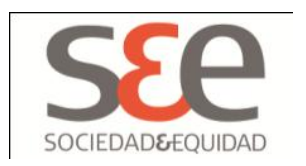

\section{Conclusiones}

El análisis de las dos fotonovelas de Corín Tellado "Te atreviste por Mî" y "Te vi Pasar" muestra que se construye, legitima y reproduce, tanto la identidad femenina como masculina en el discurso por medio de patrones culturales (o estereotipos), donde se reconoce:

1. El ideario de una mujer romántica, enamorada del amor, que concibe el matrimonio como la culminación de este y al que sólo es posible llegar en función de una simbiosis sentimental con el otro (hombre).

2. El reconocimiento y exaltación de valores en la mujer, tales como la honestidad, la humildad, la sinceridad, honradez, etc., a través de los cuales se instala la concepción de mujer perfecta que cualquier varón desearía tener como esposa.

3. El cuestionamiento social de las figuras femeninas que se alejan de los cánones establecidos para la época, vale decir, buena esposa, hacendosa y cariñosa. Sin embargo, se presentan dicotomías entre el discurso femenino políticamente correcto y el aspiracional a través del cual las mujeres buscan una cierta independencia basadas en experiencias vicarías.

4. La censura de la sexualidad femenina, ya sea por medio del autocastigo moral como social. Por tanto, el deseo y la excitación conforman el punto antagónico a la premiada pureza y virginidad de la mujer.

5. Una identidad masculina que se constituye, exaltando preferentemente valores o características negativas, es decir, el descompromiso afectivo y la irresponsabilidad, entre otros, que vienen a ser factores atentatorios contra el amor romántico femenino y el matrimonio. Cabe señalar que la instalación de la mujer ideal toma aquí relevancia, puesto que esta vendría a ordenar la vida desenfrenada de este tipo de varones.

6. La aprobación externa hacia la figura de un hombre que es capaz de controlar sus impulsos, así como el rechazo discursivo de la situación contraria.

7. La presencia de generalizaciones respecto a los varones que forman parte del imaginario social, e intimidatorias de la figura femenina, por ejemplo, el abuso de poder de estos sobre las mujeres en situaciones laborales.

8. El uso de un narrador omnipresente que cumple una doble función: por una parte, se transforma en la conciencia femenina a través de la que se expresan sentimientos $u$ emociones reprimidas en el diálogo y, por otra, viene a perpetuar los rasgos identitarios masculinos negativos transversales en el texto.

En vista de los resultados obtenidos, esta investigación abre una arista en los estudios críticos relativos a aplicaciones prácticas ilimitadas dadas no sólo por el trabajo con otros medios de comunicación masivos nacionales y extranjeros (donde se incluyan los diferentes formatos); sino 


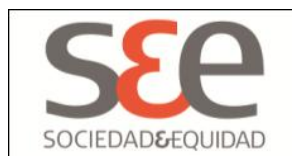

que también se incorpore la literatura, la música, los textos escolares, etc. Esto radica en que la temática de la construcción de la identidad masculina y femenina es inherente a cualquier sociedad. En todo caso, es importante señalar que este trabajo constituye una primera aproximación al fenómeno, por lo tanto, sus resultados y aspectos metodológicos requieren en el futuro ser profundizados y extrapolados a otros corpus de estudio. 


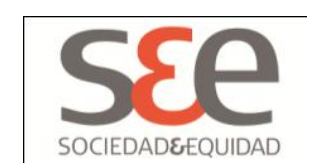

\section{Referencias Bibliográficas}

Adorno, T. (1967) La industria cultural. Buenos Aires: Galena.

Alonso, E. (2011) Cincuenta años de usos amorosos: El amor y la novela rosa. Ogigia, 9: 33-43.

Álvarez, G. (2001) Textos y discursos. Introducción a la Lingüística del Texto. Concepción: Editorial Universidad de Concepción.

Burgueño, C. (2006) Apuntes de variables sociales y ACD. Santiago: Manuscrito no publicado.

Blanco, C. y Silva, F. (1998) De mitos y quimeras. La legitimación del discurso del placer de la sexualidad en dos suplementos y una revista, desde la perspectiva del análisis crítico del discurso. Santiago: Universidad de Santiago de Chile.

Blázquez, M. (2008) Amor y sexualidades o lo que nos han contado sobre el amor como cuenta en nuestra(s) sexualidad(s). Trabajo presentado en $V$ Jornadas sobre Violencias de Género y VIH: Amor, Sexo y Otros Cuentos, Bilbao, España.

Charadeau, P. y Maingenau, D. (2005) Diccionario de análisis del discurso. Buenos Aires: Amorrortu.

Fairclough, N. (1998) Propuestas para un nuevo programa de investigación en el análisis crítico del discurso. En Rojo, M y Whittaker, R (eds.). Poder -decir o el poder de los discursos. Madrid: Arrecife.

Fairclough, N. y Wodak, R. (2001) Análisis crítico del discurso. En Van Dijk, T. (comp.). El Discurso como Interacción Social. Barcelona: Gedisa.

Farías, M. (2006) Apuntes de análisis del discurso. Santiago: Manuscrito no publicado.

Foucault, M. (1999) El orden del discurso. Barcelona: Tusquets.

Gee, J. P. (1999) An introduction to discourse analysis. Londres: Routledge.

Guerra, L. (1989) Estrategias discursivas en la narrativa de la mujer latinoamericana. Trabajo presentado en el X Congreso de la Asociación de Hispanistas. Barcelona, España.

Jaworski, A.y Coupland, N. (Eds.) (1999) Introduction: perspectives on discourse anlysis. The discourse reader. Londres: Routledge.

Martínez, E. (2000) Bildungsroman y crítica de género. Novela de rosa y narrativa de mujeres. Cuadernos de Filología Italiana, $n^{\circ}$ extraordinario, 529-546. 


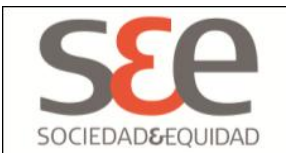

Martínez, M. (2007) La construcción de la feminidad: La mujer como sujeto de la historia y como sujeto de deseo. Actualidades en Psicología, № 21, 2007: 79-95.

Martínez-Plana, M. (2004) Entre velas y rosas: Algunas dimensiones del amor romántico. Revista de Ciencias Sociales, № 13, 2004: 8-27.

Pomerantz, A. y Fehr, B. J. (2001) Análisis de la conversación: enfoque del estudio de la acción social como práctica de producción de sentidos. En van Dijk (Comp). El discurso como interacción social. Barcelona: Gedisa.

van Dijk, T. (1982). Episodes as units of discourse analysis, en D. Tannen (Ed.) Analyzing discourse: Text and talk. Washington: GU Press.

(1997a) La ciencia del texto. Un enfoque Multidisciplinario. Barcelona: Paidós.

(1997b) Objetivos del análisis crítico del discurso. Racismos y análisis crítico de los medios. Buenos Aires: Paidós.

(2001) El estudio del discurso. En Van Dijk (Comp.). El discurso como estructura y proceso. Barcelona: Gedisa.

(2003) La multidisciplinaridad del análisis crítico del discurso. En Wodak, R. y Meyer, M. (Comp). Métodos de análisis crítico del discurso. Barcelona: Gedisa.

Vera, H., Aravena, S. y otros. (2004). Calidad de la información periodística. Investigación sobre diarios chilenos: aspectos teóricos y metodológicos. Santiago: Universidad de Santiago de Chile. 


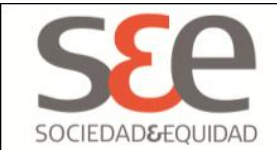

TABLA 1. CUADRO RESUMEN CORPUS

\begin{tabular}{|c|c|c|c|c|c|}
\hline \multirow{3}{*}{$\begin{array}{l}\text { CORPUS } \\
\text { FOTONOVEA }\end{array}$} & \multicolumn{4}{|c|}{ CONS TRUCCIONDELA IDENTIDAD } & \multirow{3}{*}{$\begin{array}{c}\text { TOTAL } \\
\text { ENUACADOS }\end{array}$} \\
\hline & \multicolumn{4}{|c|}{ NAFFADCR } & \\
\hline & MUER & HOMERE & MWER & HOMEFE & \\
\hline FOTONOVEA 1 & 22 & 16 & 7 & 2 & 47 \\
\hline FOTONOVEA 2 & 20 & 17 & 1 & 1 & 39 \\
\hline TOIMUIFS & 42 & 33 & 8 & 3 & 88 \\
\hline
\end{tabular}

Creado por la autora.

TABLA 2. CUADRO CONSTRUCCIÓN DE LA IDENTIDAD FEMENINA

\begin{tabular}{|c|c|c|}
\hline \multirow[b]{2}{*}{$\begin{array}{c}\text { CORPUS } \\
\text { FOTONOVELA }\end{array}$} & \multicolumn{2}{|c|}{ CONSTRUCCIONDELA IDENTIDAD } \\
\hline & MUJER & $\begin{array}{l}\text { PORCENTAJE } \\
\text { ENUNCIADOS }\end{array}$ \\
\hline FOTONOVELA 1 & 22 & $25,50 \%$ \\
\hline FOTONOVELA 2 & 20 & $23,26 \%$ \\
\hline TOTRLES & 42 & $48,80 \mathrm{~W}$ \\
\hline
\end{tabular}

Creado por la autora. 


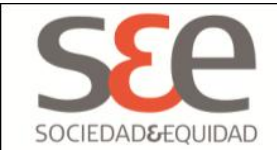

TABLA 3. CUADRO CONSTRUCCIÓN DE LA IDENTIDAD MASCULINA

\begin{tabular}{|c|c|c|}
\hline $\begin{array}{c}\text { CORPUS } \\
\text { FOTONOVELA }\end{array}$ & CONS TRUCCION DE LA IDENTIDAD & \\
\cline { 2 - 3 } & HOMBRE & $\begin{array}{l}\text { PORCENTAJE } \\
\text { ENUNCIADOS }\end{array}$ \\
\hline FOTONOVELA 1 & 16 & \\
\hline FOTONOVELA 2 & $17,60 \%$ & \\
\hline TIOTRLES & 17 & \\
\hline
\end{tabular}

Creado por la autora.

TABLA 4. CUADRO CONSTRUCCIÓN DE LA IDENTIDAD FEMENINA Y MASCULINA DESDE EL NARRADOR

\begin{tabular}{|c|c|c|c|}
\hline \multirow{3}{*}{$\begin{array}{l}\text { CORPUS } \\
\text { FOTONOVELA }\end{array}$} & \multirow{2}{*}{\multicolumn{2}{|c|}{$\begin{array}{c}\text { CONSTRUCCION DE LA IDENTIDAD } \\
\text { NARRADOR }\end{array}$}} & \multirow{3}{*}{$\begin{array}{l}\text { PORCENTAJE } \\
\text { ENUNCIADOS }\end{array}$} \\
\hline & & & \\
\hline & \begin{tabular}{|l|} 
MUJER \\
\end{tabular} & HOMBRE & \\
\hline FOTONOVELA 1 & 7 & 2 & $10,46 \%$ \\
\hline FOTONOVELA 2 & 1 & 1 & $2,31 \%$ \\
\hline TOTHLES & 8 & 3 & 127 \\
\hline
\end{tabular}

Creado por la autora. 